\title{
Using Collaborative Learning to Teach WCAG 2.0
}

\author{
Fernando Alonso, José L. Fuertes, Ángel L. González, and Loïc Martínez \\ Facultad de Informática, Universidad Politécnica de Madrid, Spain \\ \{falonso, jfuertes, agonzalez, loic\}@fi. upm. es
}

\begin{abstract}
Version 2.0 of the Web Content Accessibility Guidelines (WCAG) was published in December 2008. WCAG 2.0 has a different language, a different structure and a different rationale to WCAG 1.0. All of these influence how to teach web accessibility. In this paper we present an innovative approach that we have followed in a web accessibility module that is taught at the UPM's School of Computing as part of the BEng degree in Computer Science. Our approach combined several teaching methods: traditional lectures, collaborative learning sessions, a short exercise on web site evaluation and, finally, a short project consisting of the development of an accessible web site. In the paper we describe the methods used and the results.
\end{abstract}

\section{Introduction}

Version 2.0 of the Web Content Accessibility Guidelines (WCAG) was published in December 2008 [1]. This new version of WCAG had two main goals: it aimed to be technology-independent and it was to be testable.

Given these two goals, WCAG 2.0 has a different language, a different structure and a difterent rationale to WCAG 1.0. All of these influence how web accessibility should be taught. Here we present an innovative approach that we adopted in a web accessibility module taught at the School of Computing of the Technical University of Madrid (UPM) as part of the BEng in Computer Science.

The content of this paper is as follows. Section 2 will provide a short overview of WCAG 2.0. Section 3 will describe our teaching approach. Finally, Section 4 will provide the results and some conclusions.

\section{WCAG 2.0 Overview}

WCAG 2.0 contains three layers of guidance [1]. The four principles (perceivable, operable, understandable and robust) provide the foundation for web accessibility. Each principle contains one or more guidelines. These 12 guidelines provide the basic goals to be attained in order to make content more accessible. Testable success criteria are provided for each guideline. There are 61 success criteria with three levels of conformance: A (lowest), AA, and AAA (highest).

There is an external document that supplements WCAG 2.0: "Techniques for WCAG 2.0 " [2]. This document is "informative" and provides three additional layers of guidance. Sufficient techniques provide guidance and examples for meeting the 
guidelines using specific technologies. These are not compulsory techniques, and other techniques could be used instead. Advisory techniques can enhance accessibility, but are not considered to be sufficient techniques for some reason as explained in [2]. And common failures are examples of bad practices that cause web pages to fail to meet the success criteria.

\section{Our Teaching Approach}

At the UPM's School of Computing, we have been teaching a module on Design for All and Accessible Web Design since 2003. This module is part of the 5th year of the BEng in Computer Science [3], [4]. The module is taught over a 15-week period of the winter semester from October until February.

The first opportunity we had to introduce WCAG 2.0 was in the 2009/2010 academic year, when we changed our teaching approach. In addition to traditional lectures and a short project (developing a small accessible web site), we incorporated two more methods: collaborative learning sessions and a short exercise on accessibility evaluation.

\subsection{Lectures}

The lectures are used for the introductory lessons about Design for All, diversity, assistive technologies, standards and legislation. Guest speakers also give lectures on independent living, media accessibility and telecare services.

\subsection{Collaborative Learning: Jigsaw Sessions}

The collaborative learning technique we used was jigsaw-based sessions [5]. Jigsaw sessions involve providing students with short pieces of documentation and having them work together to learn collaboratively as follows:

1. The students are divided into three-member groups. Each group member is given a different piece of documentation.

2. Each student reads his or her piece of the documentation individually.

3. Then all the students from different groups that have read the same documentation meet together and share their views on the document.

4. Each student individually prepares a short presentation of his or her document.

5. The groups meet to share information about the different documents. Each student presents his or her document to the other group members.

6. The session ends with oral presentations of the documents. The presenters of each document are students that did not read the respective document. The group is evaluated with respect to the presentations made by their members.

The collaborative learning period in our module lasted four weeks. Every week there was one two-hour jigsaw session, plus a one-hour session to discuss the content of the collaborative sessions with the instructor. The first week centered on principles, 
guidelines and success criteria and the other three weeks focused on different sets of sufficient techniques and common failures.

\subsection{Short Exercise: Accessibility Evaluation}

This year we also introduced a short exercise, where students were asked to evaluate how accessible a web page is. All the students were given the same page to analyze and a spreadsheet template to fill in with the results of their evaluation.

The students had to evaluate all the success criteria. For each criterion, the students had to provide a value (pass, fail, not applicable), a list of techniques and failures that support that value, a short commentary explaining the value and a degree of confidence in their own evaluation. In addition they had to provide a global commentary and an estimation of the time spent on the exercise.

At the end of the exercise, a session was held to compare the results of the students and the two instructors. Then, the students were asked to explain and discuss their decisions with their colleagues in order to reach a group agreement. The goal of that session was to harmonize criteria when evaluating the accessibility of web pages.

\subsection{Short Project: Designing a Small Accessible Web Site}

At the end of the module students are expected to develop a small-sized accessible web site (around 5 pages). The students were allowed to choose the subject of the web site, and they were asked to provide some diversity of content: text, images, tables, forms... Then the students had to evaluate the accessibility of their own web site, using the same template they used for the accessibility evaluation exercise.

\section{Results and Conclusions}

The students" final grade is based on attendance of lectures and guest lectures, participation in the jigsaws, the results of the accessibility evaluation exercise, and our evaluation of their web site. Fig. 1 shows the evolution of average final grades since we started to teach this module (03/04) until this year $(09 / 10)$. The grades were ranked from 0 to 10 , where 5 is the minimum grade required to pass the subject.

This year the final grades were slightly lower than in other years. To gain some insight into the reasons for the poorer results, Table 1 shows the four components of the final grade this year.

Student grades in the jigsaw sessions were low because many did not attend all of the sessions. Actually, if we only considered the sessions they attended, the result would be 6.13 , and the resulting final grade would have been 6.33 , much closer to the grade for other years. Attendance of lectures and guest lectures was also highly variable. The results of the accessibility evaluation exercise were quite good and not very variable, although our studies suggest that WCAG 2.0 is not testable for beginners [6]. Finally, the results for the web site design were quite low. The students averaged almost three failed level-A success criteria. There were two prominent failures: $83 \%$ failed 2.4.1 (skip blocks), and $67 \%$ failed 1.3 .1 (information and relationships). Without these two mistakes, the results would have been much better. 


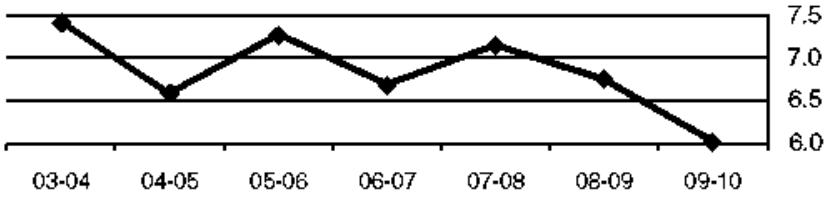

Fig. 1. Evolution of final grades

Table 1. Results for the 2009-2010 academic year

\begin{tabular}{|c|c|c|c|c|}
\cline { 2 - 5 } \multicolumn{1}{c|}{} & Attendance & Jigsaw & Evaluation ex. & Web site \\
\hline Average & 6.33 & 3.97 & 7.36 & 5.35 \\
\hline Std. deviation & 2.87 & 2.53 & 0.63 & 1.07 \\
\hline
\end{tabular}

In conclusion, this year's results were poorer, but could have been worse if we had used the old evaluation of the final grade, which did not take into account attendance, jigsaw sessions and the evaluation exercise. There are several possible reasons for this. The two key difficulties we came up against were language (WCAG 2.0 was not available in Spanish at the time) and the unavailability of evaluation tools for WCAG 2.0: the students had to perform the accessibility evaluation manually, and this led to some mistakes. In addition, we found that two success criteria were difficult to apply at design time and will require more attention in coming years.

\section{References}

1. Caldwell, B., Cooper, M., Reid, L.G., Vanderheiden, G. (eds.): Web Content Accessibility Guidelines 2.0. W3C Recommendation (2008), http: / wWw . W3 . org/TR/WCAG20/

2. Techniques for WCAG 2.0, http: / / WWW . W3 . org/TR/WCAG20-TECHS/

3. Benavídez, C., Fuertes. J.L.. Gutiérrez, E.. Martínez, L.: Teaching Web Accessibility with "Contramano" and Hera. In: Miesenberger. K. Klaus. J., Zagler. W.L., Karshmer. A.I. (eds.) ICCHP 2006. LNCS. vol. 4061, pp. 341-348. Springer, Heidelberg (2006)

4. Martínez. L.: La formación sobre accesibilidad electrónica en la UPM (in Spanish). In: European Conference EDeAN 2008, Training in Design for All: Innovative Experiences, León, Spain (2008)

5. Aronson, E., Patnoe, S.: The Jigsaw Classroom: Building Cooperation in the Classroom. Longman, New York (1997)

6. Alonso, F., Fuertes, J.L., González, A.L., Martínez, L.: On the testability of WC.AG 2.0 for beginners. In: 7th International Cross-Disciplinary Conference on Web Accessibility. ACM Press, New York (2010) 